\title{
DISTq: Low-cost, accurate and real-time estimation of insulin sensitivity
}

\author{
Paul D. Docherty*, J. Geoffrey Chase ${ }^{* *}$, Thomas F. Lotz ${ }^{* *}$, Christopher E. Hann**, \\ Geoffrey M. Shaw $* * *$, Juliet E. Berkeley****, J. I. Mann****, Kirsten McAuley***** \\ * Department of Mechanical Engineering, University of Canterbury \\ ** PhD, Department of Mechanical Engineering, University of Canterbury \\ *** MbChB, FJFICM, Department of Intensive Care, Christchurch Hospital, University of Otago, Christchurch \\ **** Dunedin School of Medicine, University of Otago
}

\begin{abstract}
Knowing insulin sensitivity $(S I)$ can optimise glycaemic control, assess metabolic drug therapy, or define diabetes risk. The DISTq is a short, low dose IM-IVGTT that generates an estimate of SI immediately after a 40 minute test using only glucose measurements, subject's physical attributes, and population parameter estimations. In this article, the DISTq is evaluated in clinical and in silics trials. In clinical trials, the test has shown a very strong correlation to the fully sampled DIST SI ( $R=0.91$ ), (which also uses insulin and c-peptide assays) and a strong correlation to the euglycemic hyperinsulinaemic clamp (EIC) in in silico virtual trials $(\mathrm{R}=0.89)$. This study shows that population estimates can reduce the need for expensive insulin and c-peptide assays in obtaining an accurate, real-time estimation of SI.
\end{abstract}

Keywords: Insulin sensitivity, diabetes risk assessment, insulin sensitivity test.

\section{INTRODUCTION}

Insulin resistance (IR) has been widely accepted as a strong indicator of an individuals risk of type 2 diabetes (T2DM) (DeFronzo \& Ferrannini 1991; Ferrannini 1997). A 10-year study of T2DM development has shown that subjects that later develop diabetes have a $60 \%$ higher IR than average. (Martin et al. 1992). IR is also a strong predictor of T2DM and of cardiovascular disease (McLaughlin et al. 2007). Accurate estimation of IR could be used to promote needed lifestyle changes that could drastically reduce the incidence of hyperglycaemia and cost associated with T2DM (Santaguida et al. 2005).

The various tests used to estimate insulin sensitivity (SI, $\mathrm{SI}=\mathrm{IR}^{-1}$ ) use various methods to provoke and measure the subject's glycaemic responses (Ferrannini \& Mari 1998; Pacini \& Mari 2003). The euglycemic hyperinsulinaemic clamp (EIC) aims to suppress endogenous glucose production (EGP) and partially suppress endogenous insulin production $\left(\mathrm{U}_{\mathrm{en}}\right)$ (DeFronzo et al. 1979). In contrast, the intravenous glucose tolerance test (IVGTT) promotes $\mathrm{U}_{\text {en }}$ (Pacini \& Bergman 1986). Hence, while the parameters derived by these tests are similar, they are not equivalent. An ideal parameter for clinical or diagnostic use would measure the efficiency of insulin to dispose of glucose to the periphery at sensible glucose and insulin concentrations.

The gold standard for SI testing is the EIC. This measures the rate of glucose disposal at basal glucose with a saturative level of insulin. The EIC is extremely accurate (DeFronzo et al. 1979), but it takes 4-5 hours and approximately 10 clinician hours (2 clinicians). A result is not guaranteed with an inexperienced clinician.
The IVGTT measures the subject's response to a $20-25 \mathrm{~g}$ intravenous (IV) glucose bolus with very frequent 1-3 minute sampling. Some protocols modify the response with a $2-3 \mathrm{U}$ IV bolus of insulin following the glucose bolus (IM-IVGTT) (Pacini \& Mari 2003). SI is obtained using the minimal model (Bergman et al. 1979). The boluses in this test tend to be supra-physiological and the trial takes 2-3 hours.

Cheaper surrogate tests include fasting glucose, 2-hour glucose (2-hr OGTT) and homeostasis model assessment (HOMA). Fasting glucose allows a diagnosis of T2DM (Tominaga 1999), but does not offer any estimates of SI. Intervention is desirable prior to impaired fasting glucose, which is a symptom of the subject's inability to maintain glycaemic homeostasis. Hence, fasting glucose is not an effective screening tool.

The 2-hr OGTT measures the subject's ability to dispose a large glucose load which is administered orally. 2 hours after the ingestion of the glucose load the blood glucose concentration is measured to allow a diagnosis of T2DM.

HOMA is a fasting insulin and glucose test. Subjects with low sensitivity will require more insulin to maintain glycaemic homeostasis. This test has a inconsistent correlation with the clamp $(\mathrm{R}=-0.19 \rightarrow \mathrm{R}=-0.82)$ (Bonora et al. 2000; Mari et al. 2001 ) and does not fully represent insulin-glucose dynamics.

The dynamic insulin sensitivity test (DIST) is a short, infrequently sampled, low dose IM-IVGTT. The test takes 3045 minutes to administer. Glucose, insulin and c-peptide data are used with a clinically validated physiological model (Lotz et al. 2005). The model and data provide parameters for $\mathrm{U}_{\mathrm{en}}$, insulin clearance rate, and SI. The test has shown good correlation to the EIC in virtual trials $(\mathrm{R}=0.99)$ (Lotz et al. 
2008), and high repeatability in a clinical pilot study $(\Delta=6 \%)$ (Lotz 2007b).

This paper presents an alternative method (DISTq - quick DIST) for solving DIST data using only glucose samples and the subjects' physical attributes (height, weight, sex, and age). Glucose samples can be assayed at the bedside, thus the sample cost is extremely low and DISTq can provide SI immediately. To achieve this, the insulin concentrations in the plasma and interstitium must be estimated using knowledge available at testing. Parameter relationships derived from clinical DIST pilot study data (Lotz 2007b) can be used to generate the required estimates. The loss of insulin and cpeptide data allows estimation of SI, but unique estimation of $\mathrm{U}_{\mathrm{en}}$ and insulin clearance is no longer possible.

\section{METHOD}

The data used in this paper was generated by the DIST test clinical pilot study conducted by (Lotz 2007a). Patient characteristics ranged from healthy lean individuals to individuals with T2DM, details can be found in Lotz (2007).

\subsection{DIST Test protocol}

Subjects fasted from $10 \mathrm{pm}$ the night before. A cannula was placed in the antecubital fossa to enable blood sampling and deliver boluses. Blood samples were taken at 0, 10, 15, 20, 25, $30,35,40,50$ minutes. A glucose bolus (50\% dextrose, $50 \%$ saline), was given to the subject immediately after the $\mathrm{t}=10$ sample. An insulin bolus (actrapid) was given immediately after the $\mathrm{t}=20$ minutes sample. Three different dosing protocols were used:

- Low dose:

$5 \mathrm{~g}$ glucose,

$0.5 \mathrm{U}$ insulin

- Medium dose: $10 \mathrm{~g}$ glucose,

$1 \mathrm{U}$ insulin

- High dose:

20 g glucose,

$2 \mathrm{U}$ insulin

The DIST pilot study consisted of 2 parts. Part 1 measured the intra-dose repeatability whereas the part 2 measured the interdose repeatability. Hence, some subjects underwent 2-3 trials at the same dose and some underwent 2-3 trials with 2 dosing protocols. Full details can be found in Lotz (2007). Blood samples were assayed for plasma glucose, insulin and Cpeptide concentrations. Glucose was analysed by an enzymatic glucose hexokinase assay (Abbott). Insulin and Cpeptide were analysed with an ECLIA immunoassay (Roche Diagnostics Elecsys, Germany).

\subsection{Physiological model equations}

The physiological model used to describe the dynamics found in the test is expressed by Equations 1-5. Equations 1 and 2 were first published in Van Cauter (1992) and enable estimation of insulin secretion from the deconvolution of cpeptide concentrations. The (Sherwin et al. 1974) model to describe insulin dynamics was simplified to Equations 3 and 4 by Lotz (2007). Equation 5 is a development of the minimal model representation of glucose (Bergman et al. 1979) by (Chase et al. 2005). In contrast to the typical use of the minimal model, Equation 5 increases the identifiably of SI by fixing the $\mathrm{p}_{\mathrm{gu}}$ term to a population average.

\begin{tabular}{|l|l|}
\hline$\dot{C}=-\left(k_{1}+k_{3}\right) C+k_{2} Y+U_{e n}$ & 1 \\
\hline$\dot{Y}=k_{1} C-k_{2} Y$ & 2 \\
\hline$\dot{I}=-n_{K} I-n_{L} \frac{I}{1+\alpha_{I} I}-\frac{n_{I}}{V p}(I-Q)+\frac{U_{e x}}{V p}+\left(1-x_{L}\right) \frac{U_{e n}}{V p}$ & 3 \\
\hline$\dot{Q}=\frac{n_{I}}{V q} I-\left(n_{C}+\frac{n_{I}}{V q}\right) Q$ & 4 \\
\hline$\dot{G}=-p_{g u}\left(G-G_{e}\right)-S I\left(G Q-G_{e} Q_{b}\right)+\frac{P}{V g}$ & 5 \\
\hline
\end{tabular}

Where: $k_{1}, k_{2}, k_{3}, n_{K}, n_{L}, n_{l}$, and $n_{C}$ are rate parameters $\left(\min ^{-1}\right)$; $\alpha_{I}$ is the liver clearance saturation coefficient $\left(\mathrm{I}^{-1}\right) ; C$ and $Y$ are plasma and interstitial compartment C-peptide (pmol. $\left.\mathrm{L}^{-1}\right) ; U_{e n}$ is the endogenous insulin production rate $\left(\mathrm{pmol} \cdot \mathrm{min}^{-1}\right)$; $I$ and $Q$ are plasma and interstitial compartment insulin (mU.L ${ }^{-1}$ ); $U_{e x}$ and $P$ are the insulin and glucose boluses (g and $\mathrm{mU}$ ); $V p$ and $V q$ are volumes of distribution (L); $x_{L}$ is the first pass liver extraction (\%); $G$ is the blood-glucose concentration (mmol.L ${ }^{-}$ $\left.{ }^{1}\right)$; $G_{e}$ and $Q_{b}$ are basal levels of the respective species; $V g$ is the volume of distribution of glucose (L); and $p_{g u}$ is the noninsulin mediated glucose disposal rate (min-1)

\subsection{Data analysis}

The DISTq method uses only glucose data from the DIST test protocol to estimate an SI. Without knowledge of insulin there is an incomplete representation of the pharmaco-kinetics of the insulin-glucose system, however, SI estimation is only possible with the subject's specific insulin concentration and rate of glucose disposal. To provide a value for SI without insulin data requires accurate prediction of insulin concentrations using population derived values.

Every full DIST test produced profiles for insulin decay and endogenous insulin production that had certain common features. The magnitude of the features varied, but the form of these features is consistent. Figure 1 shows the ten important characteristics required to define a subject's insulin concentrations during a test.
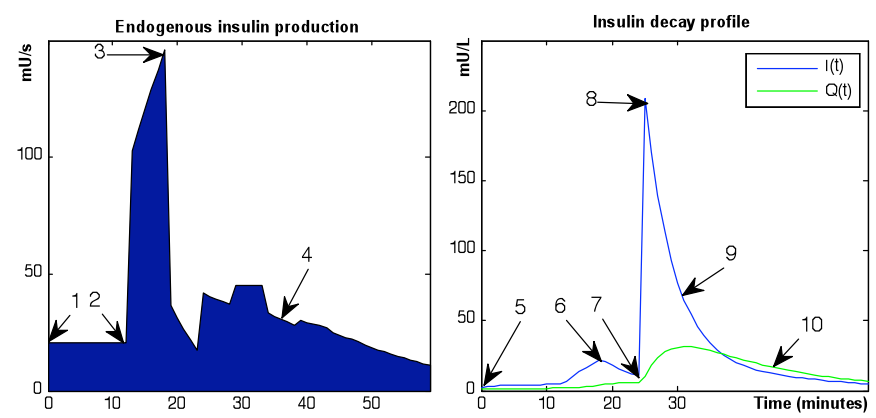

Figure 1 Unknown features that define the concentration of insulin (right), and endogenous production (left) in the subject Some characteristics can be derived from the DIST protocol or from assumptions of the full DIST model:

2.The first phase pancreatic insulin release occurs immediately after the glucose bolus (Pacini \& Mari 2003) 
6. The effect of the first phase pancreatic response on the insulin concentrations in plasma is known once the first phase response is defined. First pass liver extraction $\mathrm{x}_{\mathrm{L}}$ is set at $70 \%$ and is not a variable in this method (as it is in the full DIST methods).

7. The time of the insulin bolus is recorded per the protocol.

8. The maximum insulin concentration in the plasma is found by dividing the known bolus mass by the volume of distribution of plasma (as estimated by (Van Cauter et al. 1992)).

10. The insulin transport rate to the interstitial fluid is defined by kinetic parameters from (Van Cauter et al. 1992).

Other characteristics cannot be easily estimated:

1. Basal endogenous insulin production rate $\left(\mathrm{U}_{\mathrm{B}}\right)$.

3. The magnitude of the first phase response $\left(U_{\max }\right)$.

4. The magnitude of the second phase response $\left(U_{\text {ave }}\right)$.

5. Basal insulin concentration in the plasma $\left(\mathrm{I}_{\mathrm{B}}\right)$.

9. Liver clearance rate of insulin in plasma $\left(\mathrm{n}_{\mathrm{L}}\right)$.

The full data set from the pilot study was solved using an iterative integral method (Hann et al. 2005) and the methods described by (Lotz 2007a). The parameters that cannot be defined with only glucose data were identified and stored to aid in the generation of population-based relationships. Typically, researchers try to develop a-priori relationships between parameters (i.e. parameter $=\mathrm{f}(\mathrm{BMI}, \mathrm{BSA}, \ldots)$ ) However, no mathematical transform was apparent to linearise the relationship between BMI, or any similar a-priori metric, to these unknowns. However, when these parameters are solved for using the full DIST method and compared to SI, relationships that can be mathematically expressed are observed (Figure 2). A-posteriori identification of these unknowns with SI is reasonable.

A power relationship exists between $\mathrm{SI}$ and $\mathrm{I}_{\mathrm{B}}, \mathrm{U}_{\mathrm{B}}, \mathrm{U}_{\max }$, and $U_{\text {ave }}$. Although the relationship between SI and $U_{\max }$ is less strong, this variation is mitigated by the DISTq process. The liver clearance rate is best represented with a log relationship to SI. The mean percentage error between the measured metrics and the a-posteriori predicted metric was $36 \%$. Figure 2 and equations 6-10 show these $\mathrm{f}(\mathrm{SI})$ relationships.

\begin{tabular}{|l|c|}
\hline$u_{b}=122.49 *(S I)^{-0.7236}$ & 6 \\
\hline$u_{\max }=168.32 *(S I)^{-0.1821}$ & 7 \\
\hline$u_{\text {ave }}=177.87 *(S I)^{-0.6932}$ & 8 \\
\hline$I_{b}=63.18 *(S I)^{-1.1347}$ & 9 \\
\hline$n_{L}=0.0491 * \ln (S I)+0.0447$ & 10 \\
\hline
\end{tabular}

The DISTq method is iterative. The first iteration uses parameter estimates calculated with a population average SI of $10 \mathrm{e}^{-4} \mathrm{~L} \cdot \mathrm{mU}^{-1} \cdot \mathrm{min}^{-1}$ and Equations 6-8 to provide sufficient assumptions to generate an endogenous insulin production curve. Equations 9 and 10 along with the endogenous insulin production curve and insulin bolus knowledge provide sufficient information to simulate an insulin decay curve.
With the interstitial insulin profile generated, the real glucose data can be used to solve Equation 5 for SI. This estimated SI value allows new parameter estimations from Equations 6-10.
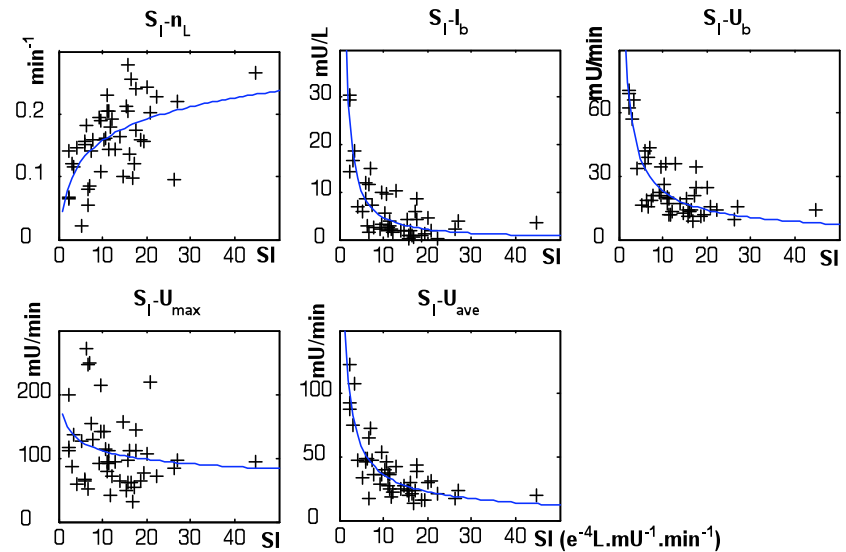

Figure 2 the a-posteriori relationships between the unknown parameters and SI which have been developed using $(\mathrm{N}=46)$ fully sampled DIST tests

The process of estimating an endogenous insulin reaction, simulation of insulin decay, and solving for SI is repeated until convergence is achieved. Typically five iterations are required.

The basic DISTq method does not fit the shape of the glucose decay. However, after a DISTq SI is found, the estimation can be improved if the high resolution of the glucose decay is utilised. To use the information in this region, $\mathrm{n}_{\mathrm{L}}$ is allowed to vary up to $20 \%$ from its population assumed value. The insulin concentration profile is re-simulated using $n_{L}$ values between $0.8 \mathrm{n}_{\mathrm{L}}$ and $1.2 \mathrm{n}_{\mathrm{L}}$, the value that allows the best glucose re-simulation fit to the measured data is used in a final estimation of SI. This is a second cycle of the algorithm. The DISTq SI is compared to the SI estimated by the full DIST data sets.

\subsection{DISTq Analysis}

To establish whether the DISTq is capable of estimating SI using only glucose data, $\mathrm{SI}_{\mathrm{DISTq}}$ will be compared to the fully sampled $\mathrm{SI}_{\text {DIST }}$ with a Pearson correlation.

A Monte Carlo simulation of an EIC cohort was designed matching the process of (Lotz et al. 2008). The simulation included a HOMA analysis as well as a full DIST and glucose only DISTq analysis. The EIC data was from 146 clamp tests (73 subject intervention study by (McAuley et al. 2002)). The Monte Carlo analysis used SI values from this cohort Lotz (2008) to run, in silico, DIST and DISTq tests with random physiological variations in sample concentration and bolus volumes (per Lotz et al. 2008). In full, 250 iterations were completed for each subject. Random assay error was added to the base data in accordance with (Lotz et al. 2008). The mean and standard deviation of SI for each trial set and solver method was stored. The coefficient of variation $(\mathrm{CV}$, $\mathrm{CV}=\mathrm{STD} /$ mean) was calculated.

The 73 subjects participated in 2 clamp tests before and after an 16 week intervention (McAuley et al. 2002). Relative changes in SI for each patient were calculated: 


$$
\Delta=\left(S I_{\text {final }}-S I_{\text {initial }}\right) / S I_{\text {initial }}(* 100 \%)
$$

The changes in SI for the real clamp and HOMA, and the virtual DIST and DISTq were thus evaluated and ranked in the order of improvement as recorded by the clamp test.

\section{RESULTS}

The DISTq showed a strong ability to replicate the SI of the fully sampled DIST test using clinically derived data. The DISTq SI showed strong equivalence with the fully sampled DIST $\left(R_{\text {pearsons }}=0.86\right)$. When the liver clearance parameter was varied in the second algorithm cycle, the correlation increased $(\mathrm{R}=0.91)$. Figure 3 shows the strength of relationship between the DIST and the DISTq.
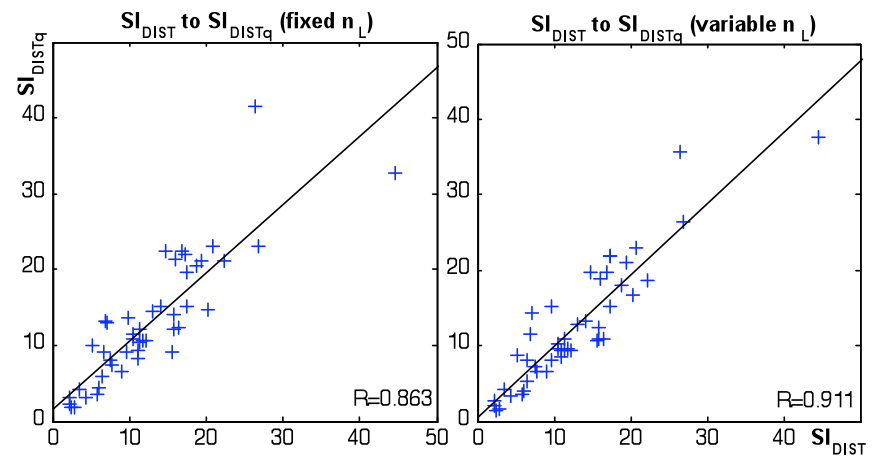

Figure 3 correlation between $\mathrm{SI}_{\text {DIST }}$ and $\mathrm{SI}_{\text {DISTq }}$ using clinically derived DIST data
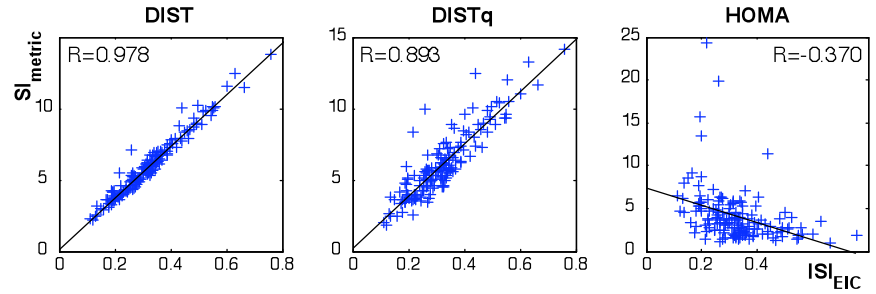

Figure 4 in-silics comparison between the DIST, DISTq and HOMA and ISIg form the clinical clamp study

Correlation between ISIg and the DIST matched Lotz et al (2008) $\mathrm{R}=0.98$. The simulation also predicted a correlation between ISIg and the DISTq was $\mathrm{R}=0.89$. The correlation between DIST and DISTq was slightly better than the clinical result, with $\mathrm{R}=0.96$. The HOMA correlation to ISIg was significantly lower at $\mathrm{R}=-0.37$.

Figure 5 confirms that the DIST and DISTq were able to accurately capture the intra-subject SI shifts with correlations to the ISIg recorded shifts of 0.97 and 0.92 respectively. HOMA showed an inability to define these shifts $(\mathrm{R}=-0.22)$.

The Monte Carlo simulation resulted in an average $\mathrm{CV}=6.6 \%$ for the full DIST, comparable to that found by (Lotz et al. 2008). The average $\mathrm{CV}=21.2 \%$ of DISTq was considerably higher. Figure 6 shows a marginal increase in $\mathrm{CV}$ at the lower end of the SI scale.
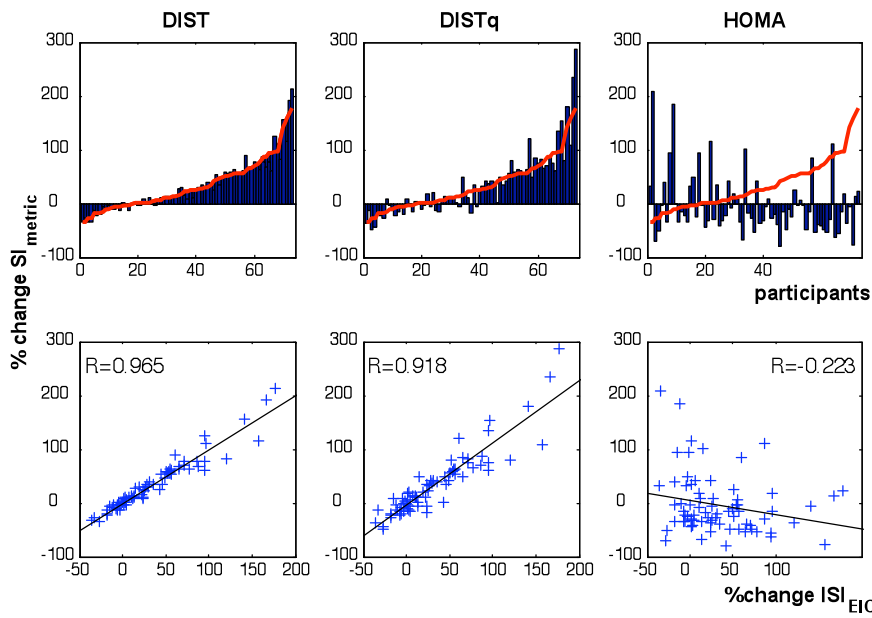

Figure 5 in-silics intra-subject shift in SI compared against ISIg

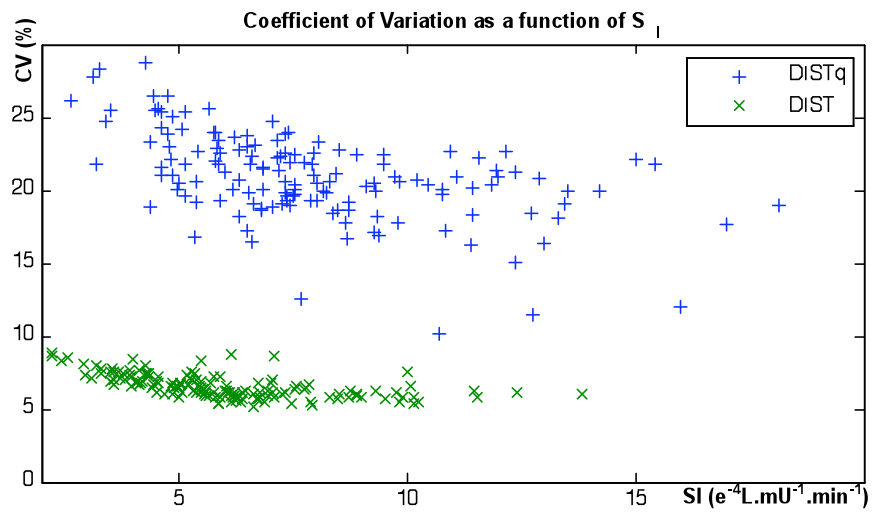

Figure 6 Coefficient of variation distribution derived by the Monte Carlo simulation

\section{DISCUSSION}

The DISTq method for estimating SI using only glucose data from the DIST protocol has shown a strong equivalence to the fully sampled DIST $(\mathrm{R}=0.86)$. This correlation can be improved by varying the liver clearance parameter in a second algorithm step $(\mathrm{R}=0.91)$. This high correlation is of significant importance, as it shows the ability to estimate SI using only glucose data and physical attributes.

DISTq can give a good estimation of SI. The method is dependant on glucose data and a series of assumptions on insulin concentration that are well justified in the literature. However, the population-derived parameter assumptions in Figures 2 may not be as valid given the smaller numbers used to derive them and resulting wider confidence bands. This weakness is likely diminished by the assumptions driven by the protocols use of insulin boluses that can mask some of the likely uncertainties. Thus, the DISTq method performs well, but greater numbers of subjects and with matching full DIST results would solidify the level of confidence in the parameter estimation step.

The DISTq CV was greatest in the low SI range due to the higher than usual variance of the unknown parameters in this region. This is evident in figure 2, particularly for the Umax metric. Newly diagnosed and long term T2DM individuals have a similar SI levels. However the endogenous insulin 
output and Umax are elevated in a newly diagnosed and diminish the long term T2DM.. This causes a trumpet in the parameter relationship data shown in figure 2 for Umax and $\mathrm{Ub}$ at low SI. More data may be required in this region to solidify our estimates.

For a new test to be accepted as a viable option, it must have a strength over tests of similar cost (in this case HOMA or OGTT). The DISTq is a very cheap test in terms of assay cost, and time; however, it is more intensive in terms of clinician activity than the OGTT or the HOMA. For DISTq to be accepted as a viable option for use it must show greater accuracy in terms of repeatability and correlation to the EIC than either the HOMA or OGTT.

Figure 4 shows that DISTq was able to detect intra-subject shifts in SI after a 3 month intervention, whereas HOMA showed a clear inability to do so. The virtually derived DISTq SI correlated well to the EIC $(\mathrm{R}=0.81)$ compared to the HOMA $(\mathrm{R}=-0.37)$. This result implies that the DIST and DISTq are more equivalent to the EIC than HOMA.

(Ferrannini et al. 2005) found a $\mathrm{R}=0.74$ correlation between the 2-hour oral-glucose tolerance test (OGTT) and the clamp which is comparable to our in silico correlation of $\mathrm{R}=0.81$. However, the reproducibility of the OGTT has been called into question by a number of researchers who found NGT, IGT and T2DM re-classification rates of only $50-65 \%$ (Ko et al. 1998; Levy et al. 1999; Park et al. 2000).

The DISTq contrasts with the OGTT by the way in which insulin and glucose are elevated in the test subject. Insulin is not measured in either test, but the DISTq protocol introduces a known amount of insulin at a set time, which can still account for the majority of the subject's plasma insulin. This approach allows strong predictions of the insulin concentration that are not possible in the OGTT protocol. A relative indifference to insulin measurements causes the metric derived by the OGTT to be more about glucose disposal than a SI. Thus, this OGTT metric can be useful in some cases, but is not ideal in clinical studies where an accurate estimation of peripheral SI is important.

The DISTq protocol requires the subject to be present for approximately an 30-60 minutes and, as the test requires an insulin bolus, must be supervised by a doctor. In contrast, HOMA is a single blood test, and the OGTT requires only a nurse to administer a glucose drink and take a blood test two hours later. Like the OGTT the DISTq can give an answer immediately after the final blood test, where HOMA requires a costly insulin assay, which may take a few days. Hence, the DISTq balances intensity, time and resolution.

The full DIST test derived SI correlates somewhat better than the DISTq to the EIC and the c-peptide assay provides unique identification of $U_{b}, U_{\max }$ and $U_{\text {average }}$ which is an indicator of beta-cell function. The subject's beta-cell function along with SI is a very strong predictor of the patients current risk in terms of the progression to type 2 diabetes (Mari et al. 2008). Hence, DIST is a stronger diagnostic but less cost efficient screening tool.
The validation of the method has been completed on the same data set that was used to derive the parameter estimation equations. Intuitively, this would increase the correlation expected between DIST and DISTq. This concern is offset when considering that the data set was a clinically derived so the predicted profiles for insulin and c-peptide are representations of actual population characteristics. The parameter relationships shown in figure 2 are noisy and no effort is taken to fit that noise. If the noise was fitted with complex multivariable equations this argument would be more valid. However, as just the general form of the relationships is developed, validating with the derivation set is valid for this first analysis.

The DISTq is likely to be a useful test where a working knowledge of the insulin sensitivity of a subject is required quickly. This situation can arise in an intensive care situation when optimal glycaemic regulation (Shaw et al. 2006) or an indicator of sepsis (Blakemore et al. 2008) is desired. As it is inexpensive and relatively accurate, it may also prove to be a suitable screening tool for the assessment of diabetes risk (Harris et al. 2003) or for analysing sensitivity changes in a drug or intervention study. It could also be used with newly diagnosed diabetic individuals to understand their current sensitivity to better control their condition with insulin.

\section{CONCLUSION}

When choosing an insulin sensitivity test the clinician or researcher should decide what level of resolution, intensity, cost, complexity, speed and confidence is best for that particular application or study. The DISTq is not an answer for all of these purposes, but should be considered when a low intensity, very inexpensive, but relatively accurate estimation of SI is desired. The low cost and intensity may allow greater numbers to be studied or tested. The test requires advanced mathematical processes, but once this is provided, the data can be analysed in real-time and the researcher or clinician can thus get a result immediately.

\section{REFERENCES}

Bergman, RN, Ider, YZ, Bowden, CR \& Cobelli, C 1979, 'Quantitative estimation of insulin sensitivity', $A m J$ Physiol, vol. 236, no. 6, pp. E667-77.

Blakemore, A, Wang, SH, Le Compte, AJ, Shaw, GM, Wong, J, Lin, J, Lotz, T, Hann, CE \& Chase, JG 2008, 'Modelbased insulin sensitivity as a sepsis diagnostic in critical care', Journal of Diabetes Science and Technology, vol. 2, no. 3, pp. 468-77.

Bonora, E, Targher, G, Alberiche, M, Bonadonna, RC, Saggiani, F, Zenere, MB, Monauni, T \& Muggeo, M 2000, 'Homeostasis model assessment closely mirrors the glucose clamp technique in the assessment of insulin sensitivity', Diabetes Care, vol. 23, no. 1, pp. 57-63.

Chase, JG, Shaw, GM, Lin, J, Doran, CV, Hann, C, Robertson, MB, Browne, PM, Lotz, T, Wake, GC \& Broughton, B 2005, 'Adaptive bolus-based targeted glucose regulation of hyperglycaemia in critical care', Med Eng Phys, vol. 27, no. 1, pp. 1-11.

DeFronzo, RA \& Ferrannini, E 1991, 'Insulin resistance. A multifaceted syndrome responsible for NIDDM, obesity, 
hypertension, dyslipidemia, and atherosclerotic cardiovascular disease', Diabetes Care, vol. 14, no. 3, pp. 173-94.

DeFronzo, RA, Tobin, JD \& Andres, R 1979, 'Glucose clamp technique: a method for quantifying insulin secretion and resistance', Am J Physiol, vol. 237, no. 3, pp. E214-23.

Ferrannini, E 1997, 'Insulin resistance is central to the burden of diabetes', Diabetes Metab Rev, vol. 13, no. 2, pp. 81-6.

Ferrannini, E, Gastaldelli, A, Miyazaki, Y, Matsuda, M, Mari, A \& DeFronzo, RA 2005, 'beta-Cell function in subjects spanning the range from normal glucose tolerance to overt diabetes: a new analysis', J Clin Endocrinol Metab, vol. 90 , no. 1, pp. 493-500.

Ferrannini, E \& Mari, A 1998, 'How to measure insulin sensitivity', J Hypertens, vol. 16, no. 7, pp. 895-906.

Hann, CE, Chase, JG, Lin, J, Lotz, T, Doran, CV \& Shaw, GM 2005, 'Integral-based parameter identification for long-term dynamic verification of a glucose-insulin system model', Comput Methods Programs Biomed, vol. 77, no. 3, pp. 259-70.

Harris, RH, Donahue, K, Rathore, SS, Frame, P, Woolf, SH \& Lohr, KL 2003, 'Screening Adults for Type 2 Diabetes: A Review of the Evidence for the U.S. Preventative Services Task Force', Ann. Intern. Med, vol. 138, no. 3, pp. 215-29.

Ko, GTC, Chan, JCN, Woo, J, Lau, E, Yeung, VTF, Chow, CC \& Cockram, CS 1998, 'The reproducibility and usefulness of the oral glucose tolerance test in screening for diabetes and other cardiovascular risk factors', Ann Clin Biochem, vol. 35, pp. 62-7.

Levy, JC, Morris, RJ, Hammersley, M \& Turner, RC 1999, 'Discrimination, adjusted correlation, and equivalence of imprecise tests: application to glucose tolerance', Am J Physiol, vol. 276, no. 2 pt 1, pp. E365-75.

Lotz, T 2007, High Resolution Clinical Model-Based Assesment of Insulin Sensitivity, University of Canterbury.

Lotz, T, Chase, JG, McAuley, KA, Shaw, GM, Wong, J, Lin, J, Le Compte, AJ, Hann, CE \& Mann, JI 2008, 'Monte Carlo analysis of a new model-based method for insulin sensitivity testing', Computer Methods and Programs in Biomedicine, vol. 89, pp. 215-25.

Lotz, TF, Chase, JG, Andreassen, S, Hann, CE, Lin, J, Wong, J \& McAuley, KA 2005, 'A fully identifiable physiological model of insulin kinetics for clinical applications', 12th International Conf on Biomedical Engineering (ICBME), Singapore, pp. 4-pages.

Mari, A, Pacini, G, Murphy, E, Ludvik, B \& Nolan, JJ 2001, 'A model-based method for assessing insulin sensitivity from the oral glucose tolerance test', Diabetes Care, vol. 24, no. 3, pp. 539-48.

Mari, A, Tura, A, Pacini, G, Kautzky-Willer, A \& Ferrannini, E 2008, 'Relationships between insulin secretion after intravenous and oral glucose administration in subjects with glucose tolerance ranging from normal to overt diabetes.', Diabet Med, vol. 25, no. 6, pp. 671-7.

Martin, BC, Warram, JH, Krolewski, AS, Bergman, R, Soeldner, JS \& Kahn, CR 1992, 'Role of glucose and insulin resistance in development of type 2 diabetes mellitus: results of a 25-year follow-up study', Lancet, vol. 340 , no. 8825 , pp. $925-9$.
McAuley, KA, Williams, SM, Mann, JI, Goulding, A, Chisholm, A, Wilson, N, Story, G, McLay, RT, Harper, MJ \& Jones, IE 2002, 'Intensive lifestyle changes are necessary to improve insulin sensitivity: a randomized controlled trial', Diabetes Care, vol. 25, no. 3, pp. 445-52.

McLaughlin, T, Abbasi, F, Lamendola, C \& Reaven, G 2007, 'Heterogeneity in prevalence of risk factors for cardiovascular disease and type 2 diabetes in obese individuals: impact of differences in insulin sensitivity', Archives Internationales De Physiologie De Biochimie Et De Biophysique, vol. 167, pp. 642-8.

Pacini, G \& Bergman, RN 1986, 'MINMOD: a computer program to calculate insulin sensitivity and pancreatic responsivity from the frequently sampled intravenous glucose tolerance test', Comput Methods Programs Biomed, vol. 23, no. 2, pp. 113-22.

Pacini, G \& Mari, A 2003, 'Methods for clinical assessment of insulin sensitivity and beta-cell function', Best Pract Res Clin Endocrinol Metab, vol. 17, no. 3, pp. 305-22.

Park, PJ, Griffin, S, Duffy, SW \& Wareham, NJ 2000, 'The effect of varying the screening interval on false positives and duration of undiagnosed disease in a screening programme for type 2 diabetes', J Med Screen, vol. 7, no. 2, pp. 91-6.

Santaguida, PL, Balion, C, Hunt, D, Morrison, K, Gerstein, HC, Raina, P, Booker, L \& Yazdi, H 2005, 'Diagnosis, prognosis, and treatment of impared glucose tolerance and impared fasting glucose', Evid Rep Technol Assess, vol. 128 , pp. 1-11.

Shaw, GM, Chase, JG, Wong, J, Lin, J, Lotz, T, Le Compte, AJ, Lonergan, TR, Willacy, MB \& Hann, CE 2006, 'Rethinking glycaemic control in critical illness - from concept to clinical practice change', Crit Care Resusc, vol. 8, no. 2, pp. 90-9.

Sherwin, RS, Kramer, KJ, Tobin, JD, Insel, PA, Liljenquist, JE, Berman, M \& Andres, R 1974, 'A model of the kinetics of insulin in man', $J$ Clin Invest, vol. 53, no. 5, pp. 1481-92.

Tominaga, M 1999, 'Diagnostic criteria for diabetes mellitus', Rinsho Byori, vol. 47, no. 10, pp. 901-10.

Van Cauter, E, Mestrez, F, Sturis, J \& Polonsky, KS 1992, 'Estimation of insulin secretion rates from C-peptide levels. Comparison of individual and standard kinetic parameters for C-peptide clearance', Diabetes, vol. 41, no. 3 , pp. $368-77$. 